\title{
Simulations of coherent beam-beam modes at the Large Hadron Collider
}

\author{
M. P. Zorzano and F. Zimmermann \\ CERN, SL Division (AP), CH-1211 Geneva 23, Switzerland
}

(Received 31 January 2000; published 26 April 2000)

\begin{abstract}
The transverse coherent motion of two colliding proton beams at the Large Hadron Collider is studied by multiparticle tracking. We use the beam-beam force for a Gaussian beam distribution with variable barycenters and rms beam sizes, and optionally include the effect of long-range collisions and external impedance. The simulation yields the coherent and incoherent oscillation frequencies, the emittance growth of either beam, and evidence for the existence or lack of Landau damping. For head-on collisions of beams with equal sizes, we find that the $\pi$-mode frequency lies outside of the continuum frequency spread, if the ratio of the beam-beam parameters exceeds 0.6 , in accordance with predictions. For smaller ratios of the beam-beam parameters, or if, for equal beam-beam parameters, the beam sizes are widely different, the $\pi$ mode is Landau damped. When long-range collisions are also included, undamped coherent modes do still exist outside the continuum, both with and without alternating crossing planes at two interaction points. However, separating the tunes of the two beams restores the Landau damping, provided the external impedance is sufficiently small.
\end{abstract}

PACS numbers: 29.27.Bd, 29.20.-c

\section{INTRODUCTION}

Two colliding beams exert a force on each other which is focusing for beams of opposite polarity and defocusing for those of equal polarity, as for the two proton beams at the Large Hadron Collider (LHC). Solutions of the linearized Vlasov equation suggest that in the case of one bunch per beam with equal parameters (intensity, beam size, betatron tune) two dipole coherent modes of oscillation appear: the $\sigma$ mode, whose frequency is equal to the unperturbed betatron tune, and the $\pi$ mode with a tune shift of 1.21-1.33 times the beam-beam parameter $\xi$ [1]. The exact value of the tune shift depends on the beam aspect ratio.

In addition to the two coherent modes, there is a continuum spectrum representing the incoherent oscillations of individual particles in each beam. The incoherent spectrum extends from 0 to $\xi$ for particles at large and small betatron amplitudes, respectively. Landau damping occurs for oscillation modes whose frequency lies inside this continuum band. It is less effective for the $\pi$ mode since this mode can be farther apart from the continuum. Although the beam-beam interaction itself does not lead to instabilities, unless the tune is near a resonance [2], the loss of Landau damping may result in an instability driven by any small impedance component of the vacuum chamber.

The beam-beam interaction in the LHC is complicated by a nonzero crossing angle at the collision points and the large number of bunches. These lead to about 30 longrange beam-beam collisions in each interaction region, where the two beams are not fully separated into different vacuum chambers. The parasitic collisions increase the number of coherent modes and give rise to additional tune shifts.

The question whether colliding proton beams with large and equal coherent tune shifts are stable is a concern for the LHC [3]. It is predicted that, in the case of equal beam sizes, for current ratios $r>0.6$ the frequency of the $\pi$ mode lies outside of the continuum tune spread [4]. The design current ratio is 1 . A potential cure is to decouple the oscillations of the two beams by separating their tunes [5,6]. Few theoretical predictions exist on the effect of the parasitic collisions.

In this report, we investigate the frequency spectrum of the centroid bunch motion using a multiparticle tracking code. The simulation model is described in Sec. II. Section III presents results for head-on collisions of bunches with equal tunes, considering both round and flat beams. Here, the coherent mode frequencies are compared with those predicted from the linearized Vlasov equation, and, for round beams, we study the dependence of the coherent-mode frequencies on the intensity ratio and its consequences for Landau damping and emittance growth. We also compare simulation results for different beam sizes, keeping the beam-beam parameters constant. In Sec. IV we discuss simulations including long-range collisions. In Sec. V we consider two beams with unequal tunes. Finally, in Sec. VI we study the impact of coherent tune shifts caused by the machine impedance.

\section{MODEL}

We simulate the collision of two strong proton beams. Our system of normalized variables is $x=X / \sigma_{0 X}, v_{x}=$ $\beta X^{\prime} / \sigma_{0 X}, \quad y=Y / \sigma_{0 Y}, v_{y}=\beta Y^{\prime} / \sigma_{0 Y}$, where $\sigma_{0 X}=$ $\sigma_{0 Y}=\sigma$ are the nominal horizontal and vertical rms sizes and $\beta$ is the beta function at the interaction point. The prime denotes the derivative with respect to longitudinal position $s$, so that, e.g., $X^{\prime}$ is the slope of the horizontal trajectory.

Each of the beams is represented by a set of $N$ macroparticles, whose trajectories are followed over $n$ turns, 
assuming linear betatron motion and a beam-beam collision at one or two interaction points (IPs). At the IP, each particle in the bunch experiences a deflection in the field of the counterrotating beam with barycenters at $\left(\bar{x}^{(i)}, \bar{y}^{(i)}\right)$ and squared transverse sizes $M_{x x}^{(i)}=\left\langle\left(x^{(i)}-\bar{x}^{(i)}\right)^{2}\right\rangle$, $M_{y y}^{(i)}=\left\langle\left(y^{(i)}-\bar{y}^{(i)}\right)^{2}\right\rangle$. This deflection is computed assuming that the opposing beam has a Gaussian shape.
Thus, for $M_{x x}^{(i)}>M_{y y}^{(i)}$ we apply a horizontal beam-beam kick [7]

$$
\Delta v_{x}(n)=\frac{r_{p} N_{p}^{(i)}}{\gamma} \frac{\beta}{\sigma^{2}} F_{x}\left(x-\bar{x}^{(i)}, y-\bar{y}^{(i)}, M_{x x}^{(i)}, M_{y y}^{(i)}\right),
$$

with $r_{p}$ the classical proton radius, $N_{p}^{(i)}$ the bunch population, $\gamma$ the relativistic Lorentz factor, and $F_{x}$ is given by

$$
\begin{aligned}
& F_{x}\left(x, y, M_{x x}, M_{y y}\right)=\sqrt{\frac{2 \pi}{\left(M_{x x}-M_{y y}\right)}} \operatorname{Im}\left[W\left(\frac{x+i y}{\sqrt{2\left(M_{x x}-M_{y y}\right)}}\right)-\sqrt{\frac{2 \pi}{\left(M_{x x}-M_{y y}\right)}} e^{\left[-\left(x^{2} / 2 M_{x x}\right)-\left(y^{2} / 2 M_{y y}\right)\right]}\right. \\
& \left.\times W\left(\frac{x \sqrt{M_{y y} / M_{x x}}+i y \sqrt{M_{x x} / M_{y y}}}{\sqrt{2\left(M_{x x}-M_{y y}\right)}}\right)\right],
\end{aligned}
$$

where $W$ denotes the complex error function [8] (if $M_{y y}^{(i)}>M_{x x}^{(i)}$ we substitute $x$ by $y$ on both sides of the two equations, and vice versa). The vertical beam-beam force is described by the real part of the same expression. In these maps the super index $(i)$ indicates variables of the counterrotating beam.

In the case of nearly equal horizontal and vertical squared beam sizes, $M_{x x}^{(i)} \approx M_{y y}^{(i)}$, Eq. (2) is ill defined. We then use a simpler expression for the force:

$$
F_{x}\left(x, y, M_{x x}^{(i)}, M_{y y}^{(i)}\right)=\frac{2 x}{\left(x^{2}+y^{2}\right)}\left[1-\exp \left(-\frac{x^{2}+y^{2}}{M_{x x}^{(i)}+M_{y y}^{(i)}}\right)\right] .
$$

We have performed some simulations using this formula only, even for (slightly) different vertical and horizontal spot sizes. The results for initially round beams and equal transverse tunes are qualitatively and quantitatively the same as obtained using the exact expression, Eq. (2). This seems to indicate that small variations in the relative transverse beam sizes do not much affect the coherent modes.

Denoting the horizontal betatron tune by $Q_{x}$, the linear map from one IP to the next is

$$
\left(\begin{array}{c}
x(n+1) \\
v_{x}(n+1)
\end{array}\right)=\left(\begin{array}{cc}
\cos \left(2 \pi Q_{x}\right) & \sin \left(2 \pi Q_{x}\right) \\
-\sin \left(2 \pi Q_{x}\right) & \cos \left(2 \pi Q_{x}\right)
\end{array}\right)\left(\begin{array}{c}
x(n) \\
v_{x}(n)+\Delta v_{x}(n)
\end{array}\right)
$$

for the horizontal motion. An equivalent map is applied for the vertical plane, $\left(y, v_{y}\right)$.

In the simulation, the initial coordinates $\left(x, v_{x}, y, v_{y}\right)$ for two groups of $N$ macroparticles representing the two beams are selected from a Gaussian random distribution in each variable with $\langle x\rangle=\left\langle v_{x}\right\rangle=\langle y\rangle=\left\langle v_{y}\right\rangle=0$ and $\left\langle x^{2}\right\rangle=\left\langle v_{x}^{2}\right\rangle=\left\langle y^{2}\right\rangle=\left\langle v_{y}^{2}\right\rangle=1$.

A similar multiparticle tracking model was used by Keil for the study of coherent oscillations of head-on collisions [9] and later by Matsumoto and Hirata [10]. There, good agreement was found between simulation results and analytical predictions, for the vertical motion of flat beams.

To simulate parasitic (long-range) collisions, the same model is employed. The two beams collide with a horizontal separation $L_{x}$ (in units of $\sigma_{x}$ ). There is about $90^{\circ}$ betatron phase advance between the IP and the region of long-range collisions. The actual number of parasitic crossings per side of each IP is $n_{\mathrm{par}} / 2$. The betatron phase advance between the long-range collision points on one side of the interaction region is small. The effects of long-range collisions on the two sides of an IP are roughly equivalent because the betatron phase advance of $180^{\circ}$ compensates for the opposite direction of the beambeam separation. Thus, in the worst case all the kicks experienced before and after the IP are added coherently. In the simulation, we lump all $n_{\text {par }}$ parasitic collisions into a single kick $90^{\circ}$ with $n_{\text {par }}$ times enhanced strength, applied behind the IP. This may slightly overestimate the effect of the long-range collisions, because it ignores possible phase differences between bunches [11]. A static dipole kick would induce a change in the closed orbit. In the simulation the static kick from the long-range collision must be subtracted to have the correct reference system. The long-range beam-beam kick is then

$$
\begin{aligned}
\Delta v_{x}(n)= & +n_{\mathrm{par}} \frac{2 r_{p} N_{p}^{(i)}}{\gamma} \frac{\beta}{\sigma^{2}}\left\{\frac{\left(x-\bar{x}^{(i)}-L_{x}\right)}{R^{2}}\left[1-\exp \left(-\frac{R^{2}}{M_{x x}^{(i)}+M_{y y}^{(i)}}\right)\right]\right\} \\
& -n_{\mathrm{par}} \frac{2 r_{p} N_{p}^{(i)}}{\gamma} \frac{\beta}{\sigma^{2}}\left\{-\frac{1}{L_{x}}\left[1-\exp \left(-\frac{L_{x}^{2}}{M_{x x}^{(i)}+M_{y y}^{(i)}}\right)\right]\right\}
\end{aligned}
$$


where

$$
R^{2}=\left(x-\bar{x}^{(i)}-L_{x}\right)^{2}+\left(y-\bar{y}^{(i)}\right)^{2} .
$$

An equivalent expression will be used for vertical longrange collisions with separation $L_{y}$.

In the simulations we assume the following typical LHC parameters: fractional betatron tunes of $Q_{x} \approx Q_{y}=$ 0.32 , bunch population $N_{p}^{(1)}=1.05 \times 10^{11}$ for beam 1 , and $N_{p}^{(2)}=r N_{p}^{(1)}$ for the second beam with $r$ varying between 0 and 1 , proton beam energy of $7 \mathrm{TeV}$, unperturbed horizontal and vertical rms beam sizes at the primary collision point $\sigma=16 \times 10^{-6} \mathrm{~m}$, and an IP beta function $\beta_{x, y}=0.5 \mathrm{~m}$. The beam-beam parameters are defined by

$$
\xi_{x, y}^{(i)}=\frac{N_{p}^{(i)} r_{p} \beta_{x, y}}{2 \pi \gamma \sigma_{x, y}\left(\sigma_{x}+\sigma_{y}\right)},
$$

with $i=1$ for beam 1 , and $i=2$ for beam 2 . With the above LHC parameters, we find $\xi \approx 0.0034$. For equal beam sizes, the ratio of the beam currents, $r=$ $N_{p}^{(2)} / N_{p}^{(1)}=\xi^{(2)} / \xi^{(1)}$, determines the behavior of the system [4]. The case $r=1$ is called the strong-strong limit. At the LHC, there are about 16 parasitic encounters on each side of an IP, with a minimum transverse separation of $L_{x}=7.5$ and $L_{y}=7.5$ (in units of $\sigma_{x}$ ).

\section{HEAD-ON COLLISION WITH EQUAL TUNES}

\section{A. $\pi$ and $\sigma$ modes for round beams}

We first consider the strong-strong case, $r=1$, and head-on collisions of two bunches, propagating particles through the maps in Eqs. (1) and (4), with the beambeam force $F(x, y)$ of Eq. (2). The statistical fluctuation in the macroparticle distribution is sufficient to excite the coherent modes. The transverse squared beam sizes, $M_{x x}$ and $M_{y y}$, oscillate around 1 (in units of $\sigma^{2}$ ) with a maximum deviation of $2 \%$. Hence the beams stay approximately round.

Fourier analyzing the motion of the barycenter of one bunch reveals two coupling modes. One is located at $Q$; the other has a lower frequency. In Fig. 1 the simulated frequency spectrum of one beam is plotted on a logarithmic scale, as determined by a fast Fourier transform (FFT). Here and in the following, when the frequency spectra of the horizontal and vertical centroid motion, $S_{x}(w)$ and $S_{y}(w)$, are equivalent, we depict the quadratic sum of both amplitudes, $S_{A}(w)=$ $\sqrt{S_{x}(w)^{2}+S_{y}(w)^{2}}$. The horizontal axis gives the tune shift from the unperturbed tune $Q$ in units of $\xi$.

If we Fourier analyze the centroid distances $\left(\left\langle x^{(1)}\right\rangle-\right.$ $\left\langle x^{(2)}\right\rangle$ or $\left.\left\langle y^{(1)}\right\rangle-\left\langle y^{(2)}\right\rangle\right)$, the coherent mode at the unperturbed frequency disappears. Conversely, when we Fourier analyze the sum of the centroids $\left\langle x^{(1)}\right\rangle+\left\langle x^{(2)}\right\rangle$ or $\left(\left\langle y^{(1)}\right\rangle+\left\langle y^{(2)}\right\rangle\right)$ the lower-frequency mode disappears.

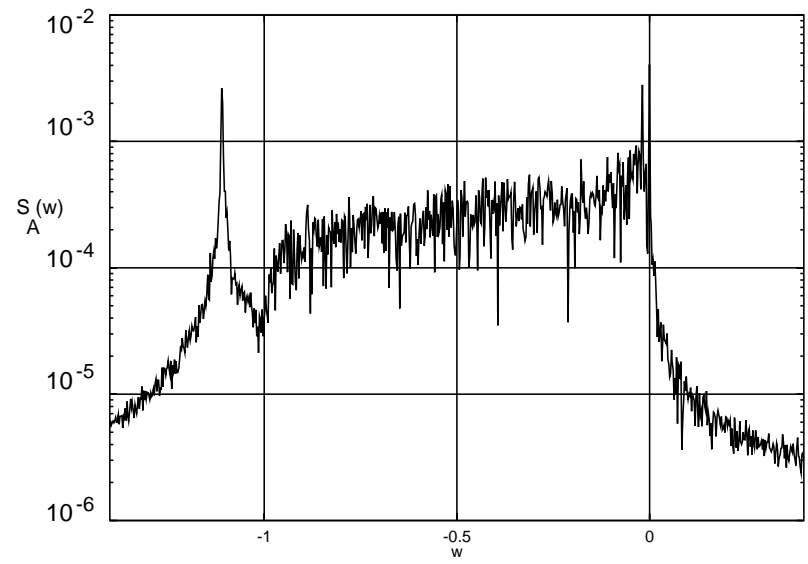

FIG. 1. Frequency spectrum of the bunch centroid motion (over $2^{17}$ turns, $N=10^{4}$ macroparticles) for round beams with $\xi_{x}=\xi_{y}=\xi=0.0034$ and $Q=0.32$. The horizontal axis gives the distance $w$ to the unperturbed tune $Q$ in units of $\xi$, i.e., $w=(\nu-Q) / \xi$. The vertical axis is the corresponding amplitude on a logarithmic scale. The $\pi$ and $\sigma$ oscillation modes are clearly visible.

We thus identify the mode at the unperturbed frequency as the so-called $\sigma$ mode, for which the centroids of the bunches oscillate in phase with equal frequencies and amplitudes. The lower frequency mode is known as $\pi$ mode. In this mode the centroids oscillate also with equal frequencies and amplitudes, but out of phase. The motion of the bunch centroids is a superposition of these two modes.

Between the $\pi$ and the $\sigma$ mode in Fig. 1 we also see the continuum. This is related to the incoherent oscillation frequencies of individual particles. A single particle of beam 1 traversing beam 2 experiences a focusing force which leads to a change in its tune. For particles near the center of the counterrotating beam this tune shift is equal to $-\xi$. For particles further away, the focusing force is smaller, due to the nonlinearity of the beam-beam force. The result is an incoherent tune spread, which extends from 0 to $-\xi$.

In our simulations the $\pi$ mode is shifted by -1.1 in units of $\xi$. Hence, the $\pi$ mode is outside of the continuum. Theoretical studies predict a tune shift between the $\sigma$ and $\pi$ modes equal to $Y \xi[1,12]$. The coefficient $Y$ describes the frequency change caused by the distortion of the beam distribution due to the beam-beam collision. This factor can be calculated from the linearized Vlasov equation, assuming a Gaussian stationary distribution. For the case of round beams this factor is predicted to be $Y=1.21$ [1]. The shift obtained in our simulations corresponds to $Y \approx 1.1$. The difference is due to either the simplifying assumptions of our model, where the beam-beam forces are calculated assuming that the beams are of Gaussian shape, or is caused by approximations in the theoretical derivation, where, e.g., the stationary distribution is assumed to be Gaussian, and the beam-beam collisions are averaged over the circumference. 

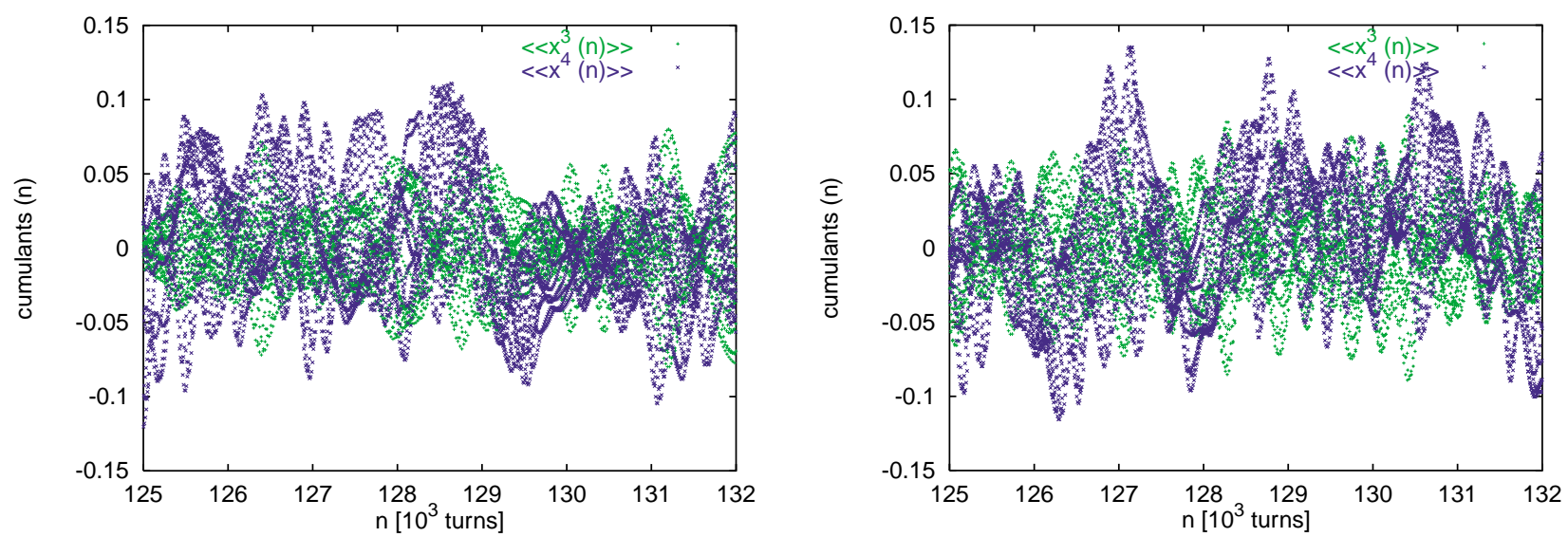

FIG. 2. (Color) Evolution of the cumulants of order 3 and 4 in the collision of two distributions of $N=10^{4}$ macroparticles, for the smallest amplitude of centroid oscillation of order $d=0.01$ (left) and for an initial offset of $d=0.1$ (right). The deviation from Gaussian shape is dominated by statistical fluctuations due to the finite number of macroparticles and is independent of the amplitude of centroid oscillation.

In a Gaussian distribution, the cumulants of order higher than 2 are exactly equal to zero. To quantify how closely our macroparticle density resembles a Gaussian we evaluate the third and fourth order cumulants $\left(\left\langle\left\langle x^{3}\right\rangle\right\rangle=\left\langle x^{3}\right\rangle-3\left\langle x^{2}\right\rangle\langle x\rangle+2\langle x\rangle^{3}\right.$ and $\left\langle\left\langle x^{4}\right\rangle\right\rangle=\left\langle x^{4}\right\rangle-$ $4\left\langle x^{3}\right\rangle\langle x\rangle-3\left\langle x^{2}\right\rangle^{2}+12\left\langle x^{2}\right\rangle\langle x\rangle^{2}-6\langle x\rangle^{4}$ ) for two different initial oscillation amplitudes. First, we consider the natural amplitude of centroid oscillation due to the finite
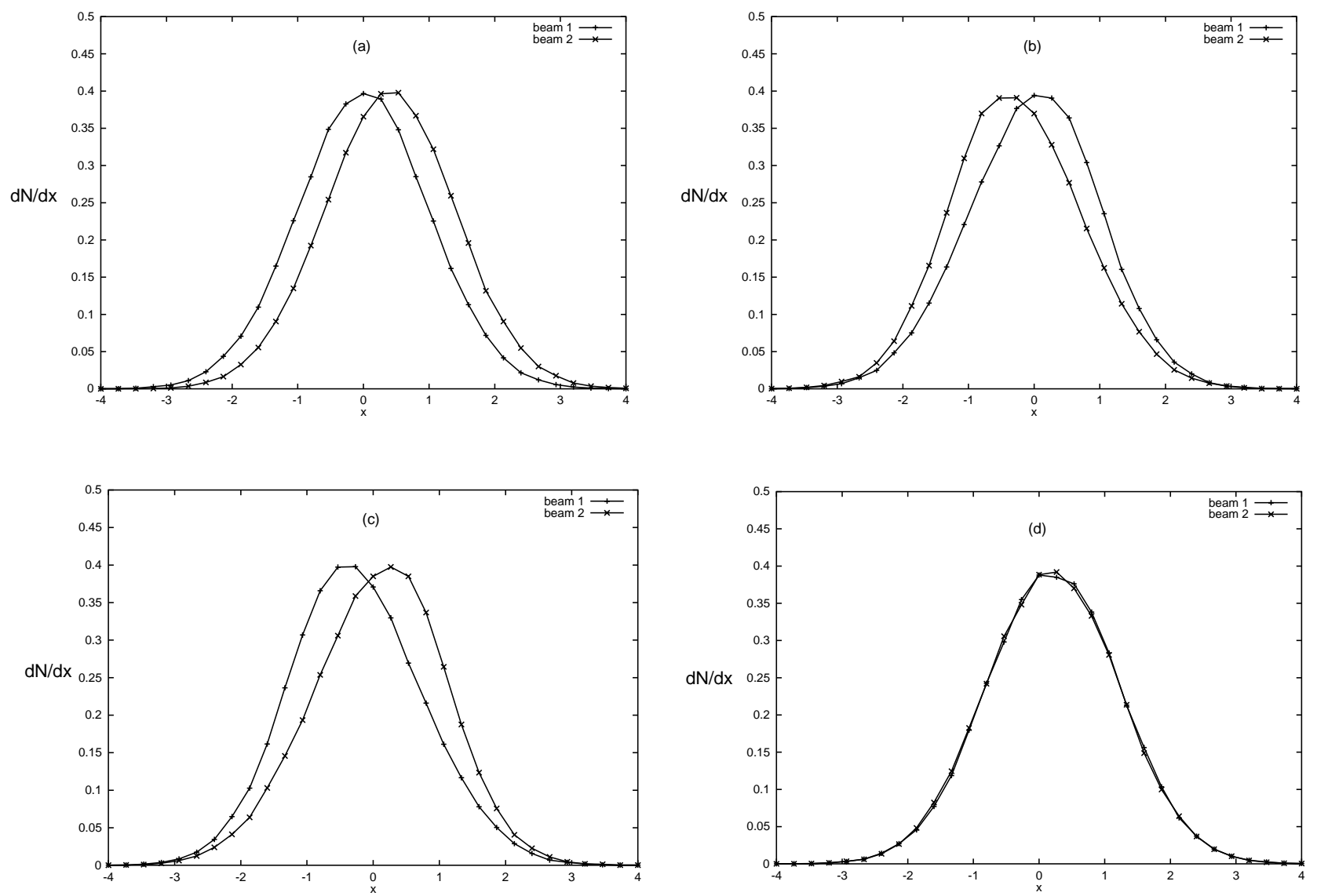

FIG. 3. Dynamics of the two strong beams $\left(N=10^{5}\right.$ macroparticles, $\left.Q=0.32\right)$ in a one-dimensional simulation for round beams. The horizontal axis is the normalized position $x$. The vertical axis shows the macroparticle distribution as obtained from the simulation. (a) Initial state, (b) after $2^{17}-2$ turns, (c) after $2^{17}-1$ turns, (d) after $2^{17}$ turns. The core of the beam oscillates coherently and the tails do not move. 
number of macroparticles. The simulation uses $N=10^{4}$ particles per bunch, with a Gaussian distribution with standard deviation of 1 , which gives an rms spread in the centroid position of $1 / \sqrt{N}=0.01$. For this amplitude of centroid oscillation, $\left\langle\left\langle x^{3}\right\rangle\right\rangle$ and $\left\langle\left\langle x^{4}\right\rangle\right\rangle$ oscillate from turn to turn around zero with rms value, evaluated over 6600 turns, $\left\langle\left\langle x^{3}\right\rangle\right\rangle_{\mathrm{rms}}=0.023$ and $\left\langle\left\langle x^{4}\right\rangle\right\rangle_{\mathrm{rms}}=0.046$. When the centroid oscillation is excited by an initial horizontal offset of $d_{x}=0.1 \sigma$, the rms cumulants are about the same $\left\langle\left\langle x^{3}\right\rangle\right\rangle_{\mathrm{rms}}=0.029$ and $\left\langle\left\langle x^{4}\right\rangle\right\rangle_{\mathrm{rms}}=0.042$. In Fig. 2 we show the turn by turn evolution of the cumulants of order 3 and 4 over 6600 turns for these two cases. The instantaneous deviation from Gaussian shape is of the order of a few percent, independent of the amplitude of the centroid oscillation. We conclude that, for small centroid oscillation amplitudes, as considered here, the deviation from the Gaussian shape is dominated by statistical fluctuations due to the finite number of macroparticles. For this number of macroparticles and small offsets, it would be difficult to correctly represent any dynamic deformation of the bunch distribution and to go beyond the Gaussian approximation.

To study in more detail the dynamic changes of the bunch distribution, we consider an even larger offset of 0.4 (in units of $\sigma$ ), which strongly excites the coherent modes in a one-dimensional simulation over $2^{17}$ turns. Figure 3(a) depicts the initial Gaussian distribution of the two beams, while the other three pictures in Fig. 3 show the distribution on the last three of $2^{17}$ turns. Comparing 3(c) with the initial distribution in 3(a) reveals that, primarily, the core of the beam participates in the oscillation.

The beam-beam interaction can also induce higherorder coherent modes. For the quadrupole mode a tune shift of $\Delta_{\pi}=-2 \times 1.022 \xi$ is predicted. The frequencies of the higher modes are even closer to the continuum. Thus, these modes are more easily Landau damped than the dipole mode, which could explain why, in the simulation, we have not observed coherent quadrupole oscillations. Note that the quadrupole-mode continuum ranges from $2 Q$ to $2 Q-2 \xi$.

The calculations of this section have been repeated using the round-beam formula for the beam-beam kick, Eq. (3), instead of Eq. (2). The results are indistinguishable from those shown here.

We have also checked the dependence on the working point. The size variations as a function of tune are in good agreement with the dynamic-beta effect. The factor $Y$ also changes slightly. These results have been reported in [13].

\section{B. $\pi$ and $\sigma$ modes for flat beams}

The tune shift of the coherent $\pi$ mode depends on the vertical-to-horizontal aspect ratio at the collision point. Simulating the collision of two flat beams with $\sigma_{x}=$ $16 \sigma_{y}$ (which is a rather typical parameter for $e^{+} / e^{-}$ storage rings) and $N_{b}=1.01 \times 10^{13}$ (we artificially increased the number of particles per bunch in order to maintain a large $\xi$ parameter and a large frequency spread), we find a vertical coherent $\pi$ mode with a tune shift of $-1.09 \xi_{y}$ and a horizontal tune shift of $-1.15 \xi_{x}$. In the case of very flat beams the linearized Vlasov theory predicts a tune shift of $-1.24 \xi_{y}$ for the vertical $\pi$ mode and $-1.33 \xi_{x}$ for the horizontal $\pi$ mode. Figure 4 shows the spectrum of the centroid motion of one of the two beams.

Similar simulations were presented in Ref. [14] for the collision of $e^{+} / e^{-}$following the evolution of 500 macroparticles over 500 turns. There, for $Q_{x}=0.25$, $Q_{y}=0.3$, and $\xi_{y}=0.0306$, a vertical $\pi$-mode tune shift of $1.06 \xi_{y}$ was obtained.

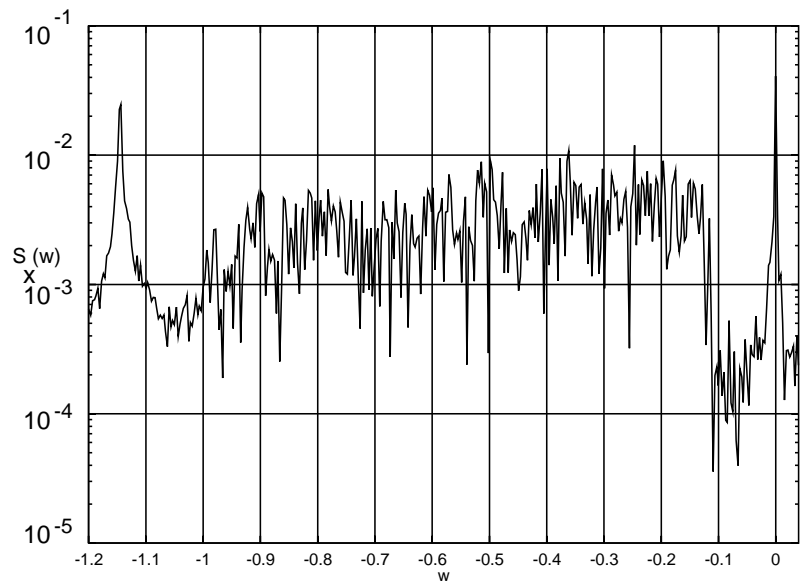

FIG. 4. Frequency spectrum of the vertical (left) and horizontal (right) bunch centroid motion for flat beams (calculated over $2^{17}$ turns, with $N=10^{4}$ macroparticles) for $\xi_{y}=0.03691$ and $\xi_{x}=0.002466$. The horizontal axis is the distance to the unperturbed tune in units of the corresponding beam-beam parameter; the vertical axis gives the oscillation amplitude on a logarithmic scale. The tune shift of the $\pi$ mode with respect to the $\sigma$ mode is different in the two planes. 


\section{Landau damping}

The case of interest for proton colliders is the round beam limit. From now on we will consider only round beams.

In the $\sigma$ mode the beams oscillate in phase at the IP without changing their shape. Participating in the $\pi$ mode are mainly particles with small betatron amplitudes. These are most strongly affected by the movements of the opposing beam, which may explain the large value of the coherent tune shift [1,4]. In the limit $r \rightarrow 0$ such a mode does not exist (unlike the discrete $\sigma$ mode which exists at any value of the intensity ratio). Therefore, for decreasing intensity of one of the beams there is a point at which the discrete $\pi$ mode disappears. Once the frequency of the $\pi$ mode lies within the incoherent tune spread its energy is absorbed by individual particles with similar oscillation frequencies. This phenomenon is known as Landau damping. As a consequence, an initial $\pi$-mode oscillation will disappear and the beam emittance will grow until the $\pi$-mode energy has been completely absorbed.

The disappearance of the $\pi$ mode is studied by varying the parameter $r$. We launch the two beams with an initial horizontal offset $d=0.2$ (in units of $\sigma_{x}$ ). The frequency spectrum for an intensity ratio $r=1$ is shown in Fig. 5. The figure depicts the amplitudes of both $\sigma$ and $\pi$ modes, obtained from an FFT of the beam centroid sum and difference, respectively. The $\pi$ mode is outside the continuum and not damped. Figure 6, for $r=0.6$, compares the frequency spectrum of the centroid distance ( $\pi$ mode) at two consecutive time intervals of $2^{16}$ turns, demonstrating that the amplitude of the $\pi$ mode decreases

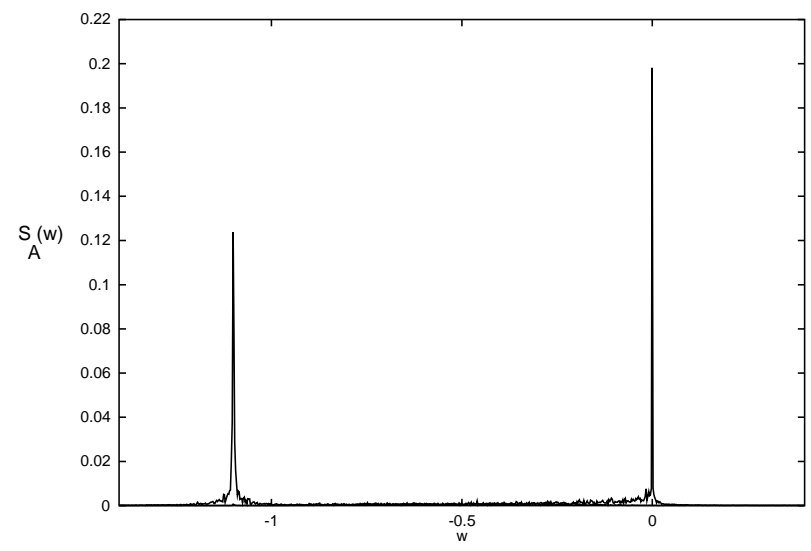

FIG. 5. Frequency spectrum of the centroid motion (over $2^{17}$ turns, $N=10^{4}$ ) for the case of round beams colliding headon with an initial offset of $d=0.2$ (in units of $\sigma_{x}$ ). The ratio of the currents is equal to $r=1$. The horizontal axis gives the tune shift from the unperturbed tune $Q$ in units of $\xi$, i.e., $w=(\nu-Q) / \xi$. The vertical axis is the corresponding amplitude obtained from an FFT, on a linear scale. The $\pi$ mode at $w=-1.1$ is not damped and its amplitude is constant over this time.

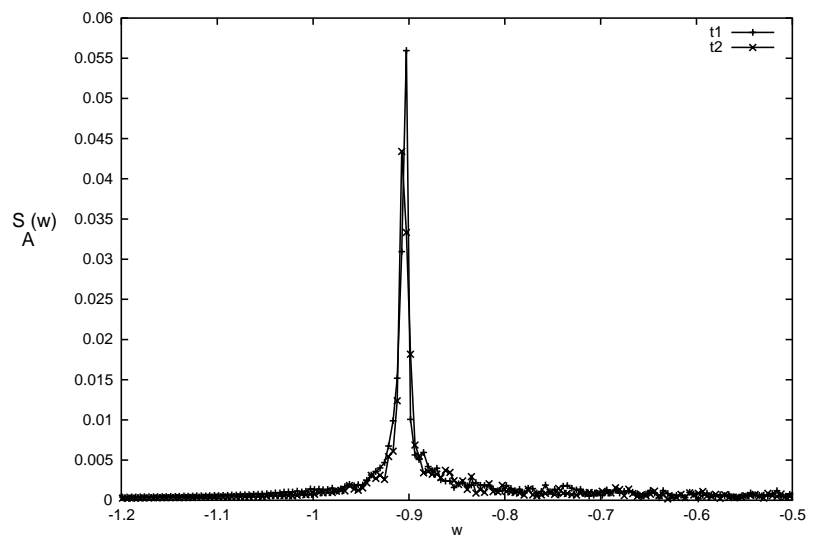

FIG. 6. Frequency spectrum of the centroid motion at two successive time intervals of $2^{16}$ turns $\left(N=10^{4}\right)$, considering head-on collisions of two round bunches with current ratio $r=0.6$ and initial offset of $d=0.2$ (in units of $\sigma_{x}$ ). The $\pi$ mode at -0.9 is Landau damped. Solid line and plus symbols: first time interval; dashed line and crosses: second interval.

in time. Since for $r=0.6$ the $\pi$-mode frequency lies near the edge of the continuum, it is marginally Landau damped. For the case $r=0.3$ (not shown), the $\pi$ mode is well inside the continuum and rapidly damped. These results confirm the prediction that for current ratios $r \leq$ 0.6 the $\pi$-mode frequency falls in the incoherent tune spread of the weaker beam [4].

\section{Emittance growth}

The kinetic energy of a kick is distributed among the different oscillation modes: the coherent $\sigma$ mode, the coherent $\pi$ mode, and the incoherent oscillation spectrum. The fraction of energy which is absorbed by the continuum leads to an irreversible emittance growth. In simulations for different intensity ratios $r$, we studied the emittance growth and energy distribution after applying a horizontal kick.

For comparison with analytical results, we introduce several emittancelike quantities. We characterize the total kinetic oscillation energy by generalized "emittances," defined as

$$
\boldsymbol{\epsilon}_{x}^{(i)}=\left\langle x^{2}+v_{x}^{2}\right\rangle, \quad \epsilon_{y}^{(i)}=\left\langle y^{2}+v_{y}^{2}\right\rangle,
$$

where the angular brackets denote an average over the distribution of bunch $i$. The initial (unperturbed) bunch emittance is $\epsilon_{0}=\epsilon_{x}+\epsilon_{y}=2+2=4$. After applying a horizontal kick, the beams start to oscillate coherently. A fraction of this energy is absorbed by the continuum leading to a growth of the emittances. The incoherent or intrinsic emittance is estimated by subtracting the centroid motion:

$$
\begin{aligned}
& \epsilon_{x}^{I(i)}=\left\langle(x-\bar{x})^{2}+\left(v_{x}-\bar{v}_{x}\right)^{2}\right\rangle, \\
& \epsilon_{y}^{I(i)}=\left\langle(y-\bar{y})^{2}+\left(v_{y}-\bar{v}_{y}\right)^{2}\right\rangle,
\end{aligned}
$$


Without correlation between position and velocity (i.e., $\left\langle x v_{x}\right\rangle=0$ and $\left\langle y v_{y}\right\rangle=0$ ), and if the dynamic beta effect is small, this definition agrees with the usual definition of emittance

$$
\epsilon_{x}=\left[\left\langle(x-\bar{x})^{2}\right\rangle\left\langle\left(v_{x}-\bar{v}_{x}\right)^{2}\right\rangle-\left\langle x v_{x}\right\rangle^{2}\right]^{1 / 2},
$$

except for a factor of 2 .

The oscillation spectrum for $r=1$ and an initial offset $d=0.2$ is shown in Fig. 5. The energy is distributed among the $\sigma$ mode of amplitude $A=0.2$, the $\pi$ mode of approximate amplitude $B \approx 0.13$ (notice the broad base of the $\pi$-mode peak), and the continuum. The fraction of energy absorbed by the continuum leads to an irreversible emittance growth. The relative increment of emittance is

$$
\frac{\Delta \epsilon^{I}}{\epsilon_{0}}=\left[\frac{\left(\epsilon_{x}^{I,(1)}+\epsilon_{y}^{I,(1)}\right)}{\epsilon_{0}}+\frac{\left(\epsilon_{x}^{I,(2)}+\epsilon_{y}^{I,(2)}\right)}{\epsilon_{0}}-2\right] .
$$

For $r=1$, the relative irreversible emittance growth is $\Delta \epsilon^{I} / \epsilon_{0} \approx 0.004$, as illustrated by the lower curve in Fig. 7.

For $r \leq 0.6$ the frequency of the $\pi$ mode lies in the continuum and is Landau damped. The emittance grows until the energy of this mode is completely absorbed. Examples are the two upper curves in Fig. 7. As expected, the final emittance is larger than for $r=1$, namely, $\Delta \epsilon^{I} / \epsilon_{0} \approx 0.01$.

These simulation results can be compared with analytical predictions. The beam response to a kick can be calculated from the linearized Vlasov equation [4], and the predicted emittances after a horizontal kick of magnitude $d$ (in units of $\sigma_{x}$ ) are [4]

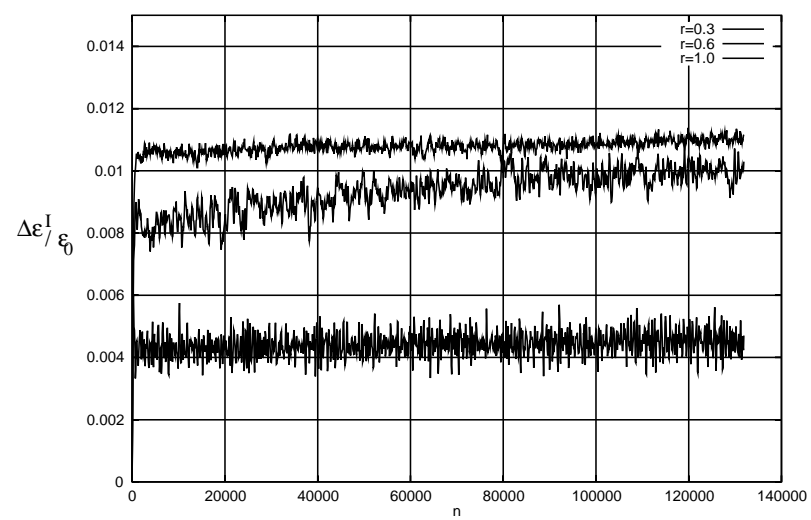

FIG. 7. Irreversible emittance growth $\Delta \epsilon^{I} / \epsilon_{0}$ (vertical axis) as a function of time (horizontal axis, time in turns) for three different current ratios, $r=0.3, r=0.6$, and $r=1$. The beams are perturbed by an initial offset of $d=0.2$ (in units of $\sigma$ ). For $r \leq 0.6$ the frequency of the $\pi$ mode lies in the continuum, and, thus, the mode is Landau damped, and the intrinsic emittance grows until the $\pi$-mode energy has been fully absorbed. For $r=1$ the $\pi$ mode is not Landau damped and carries part of the kick energy. The emittance growth is significantly smaller.

$$
\begin{aligned}
\frac{\epsilon_{x}^{(1)}+\epsilon_{y}^{(1)}}{\epsilon_{0}}+\frac{\epsilon_{x}^{(2)}+\epsilon_{y}^{(2)}}{\epsilon_{0}} & \\
& =2+\frac{d^{2}}{2}(0.5+0.32+0.18) .
\end{aligned}
$$

The first term in the brackets describes the fraction of energy carried by the $\sigma$ mode, the second represents the energy of the $\pi$ mode, and the last is the fraction which is imparted on the continuum leading to an irreversible emittance growth. The $\sigma$ mode, with amplitude $A$, carries an energy $A^{2}=\epsilon_{0} \frac{d^{2}}{2} 0.5$. The energy of the $\pi$ mode with amplitude $B$ is $B^{2}=\epsilon_{0} \frac{d^{2}}{2} 0.32$, and the relative irreversible emittance growth is $\Delta \epsilon^{I} / \epsilon_{0}=\frac{d^{2}}{2} 0.18$.

For $d=0.2$ we then expect $A=0.2, B=0.16$, and $\Delta \epsilon^{I} / \epsilon_{0}=0.0036$, in good agreement with the oscillation amplitudes shown in Fig. 5 and with the emittance growth for the lower curve in Fig. $7(r=1)$. For $r \leq 0.6$, the energy of the $\pi$ mode is transferred to the continuum. The expected relative emittance growth is $\Delta \epsilon^{I} / \epsilon_{0}=$ $\frac{d^{2}}{2}(0.18+0.32)=0.01$, again in good agreement with the simulation results of Fig. 7 (the two upper curves).

\section{E. Beam size ratio}

We have seen that for equal beam sizes the frequency of the $\pi$ mode moves out of the continuum for current or beam-beam parameter ratios $r>0.6$, as expected from the linearized Vlasov equation [4]. Experience from the SPS collider indicates, however, that factors other than the ratio of beam-beam parameters may determine the coherent stability or instability of the colliding beams [15]. When the SPS operated as a $p \bar{p}$ collider with $\xi^{(2)} / \xi^{(1)} \approx 0.8$ the $\pi$ mode was never observed. Table I lists some typical parameters for $1988 p \bar{p}$ runs [15]. The symbol $N_{b}$ is the number of particles, while $\epsilon_{h}$ and $\epsilon_{v}$ are the normalized emittances (with $\sigma$ in $\mathrm{mm}$ ) in the horizontal and vertical plane, respectively. The beams collided in two physics interaction points $\left(\beta_{h}=1 \mathrm{~m}\right.$, $\beta_{v}=0.5 \mathrm{~m}$ ) and one mid-arc interaction where $\beta \approx$ $40 \mathrm{~m}$. The fractional part of the tune was roughly 0.681 . For these parameters the ratio of beam-beam parameters in both planes was $\xi^{(2)} / \xi^{(1)} \approx 0.8$, and the beam sizes of the proton and antiproton beams differed by about $50 \%$.

Can we reproduce the absence of the $\pi$ mode in our simulation? We first simulate the head-on collision of two round bunches at one interaction point with $\beta_{h}=\beta_{v}=$ $0.5 \mathrm{~m}$ and beam-beam parameter ratio 0.8. As shown in Fig. 8 (left), with $r=0.8$ we can clearly distinguish the $\sigma$ and $\pi$ mode. Next, we simulate the collision of two bunches assuming the same ratio of beam-beam parameters, $\xi^{(2)} / \xi^{(1)}=0.8$, but unequal sizes $\sigma_{x}^{(2)}=a \sigma_{x}^{(1)}$, $\sigma_{y}^{(2)}=a \sigma_{y}^{(1)}$ with $a=1.5$ (the current of bunch $2^{x}$ is scaled as $N_{p}^{(2)}=r a^{2} N_{p}^{(1)}$, in order to maintain a constant ratio of beam-beam parameters). The right-hand picture 
TABLE I. Typical parameters at the start of a $1988 p \bar{p}$ run in the SPS. The ratio of beam-beam parameters in the two planes was $\xi^{(2)} / \xi^{(1)} \approx 0.8$; the beam-size ratio was roughly $a=1.5$.

\begin{tabular}{ccccccccccccc}
\hline \hline Parameter & $p$ & $\bar{p}$ & \multicolumn{1}{c}{$p$} & $\bar{p}$ & \multicolumn{1}{c}{$p$} & $\bar{p}$ & $p$ & $\bar{p}$ & $p$ & $\bar{p}$ & $p$ & $\bar{p}$ \\
\hline$N_{b}\left[10^{10}\right]$ & 10.9 & 3.8 & 10.1 & 4.2 & 10 & 4.6 & 9.8 & 4.6 & 10.8 & 4.4 & 9.7 & 3.8 \\
$\epsilon_{h}$ & 4.5 & 2 & 3.8 & $\ldots$ & 2.6 & $\ldots$ & 2.6 & $\ldots$ & 2.6 & $\ldots$ & 2.6 & $\ldots$ \\
$\epsilon_{v}$ & 2.7 & 1.2 & 2.3 & 1.3 & 2.3 & 1.3 & 2.4 & 1.4 & 2.4 & 1.4 & 2.3 & 1.2 \\
\hline \hline
\end{tabular}
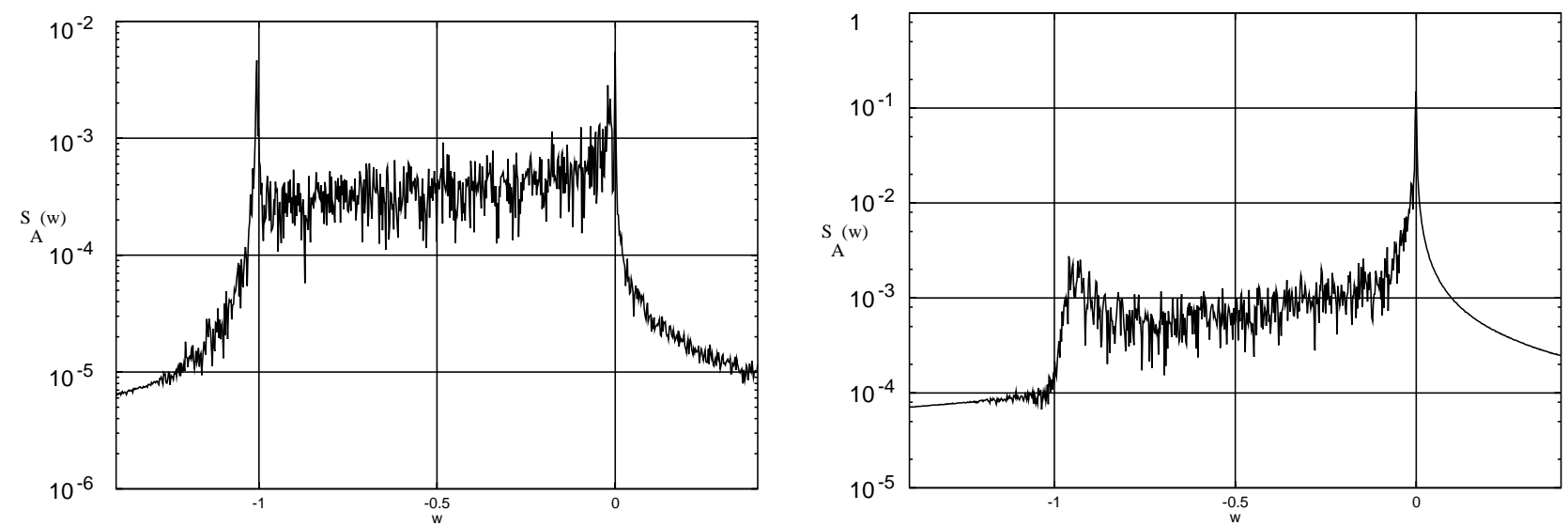

FIG. 8. Two-dimensional strong-strong simulation with $\xi^{(2)} / \xi^{(1)}=0.8$. Spectral density of centroid motion on a logarithmic scale, as a function of the distance to the nominal tune in units of $\xi^{(1)}$. Left: $N_{p}^{(2)}=r N_{p}^{(1)}, \sigma_{x}^{(2)}=\sigma_{x}^{(1)}=1, \sigma_{y}^{(2)}=\sigma_{y}^{(1)}=1$. Right: $N_{p}^{(2)}=r a^{2} N_{p}^{(1)}, \sigma_{x}^{(2)}=a \sigma_{x}^{(1)}, \sigma_{y}^{(2)}=a \sigma_{y}^{(1)}, a=1.5$ (parameters similar to the $1988 \mathrm{~S} p \bar{p} \mathrm{~S}$ run). If the beams sizes are sufficiently unequal, the $\pi$ mode disappears.

in Fig. 8 shows that in this case the $\pi$ mode (on the left of the spectrum) is absorbed by the continuum.

These results suggest that the beam-beam parameter ratio alone does not determine the location of the $\pi$ mode with respect to the continuum. A heuristic explanation of the beam-size effect is the following. Primarily, particles with small betatron amplitudes participate in the $\pi$-mode oscillation, because they are most strongly affected by the movements of the opposing beam. In the second simulation, where the size of beam 2 is increased, the fraction of particles affected by the oscillation of the counterrotating beam is smaller. Since fewer particles take part in the coherent oscillation, Landau damping is more easily established.

The LHC design requires equal beam sizes and currents $(r=1)$. In the following we will concentrate on this case.

\section{LONG-RANGE COLLISIONS}

In this section we investigate the role of the long-range collisions, both with crossing in one plane only and with alternating crossing planes at two IPs. The vertical and horizontal tunes are chosen equal $\left(Q_{x}=Q_{y}=0.32\right)$.

\section{A. Horizontal crossing (no head-on collisions)}

Figure 9 shows the horizontal and vertical spectrum of centroid oscillation of a bunch undergoing long-range collisions with a horizontal separation of $L_{x}=7.5$ (in units of $\sigma_{x}$ ). In the horizontal plane, the spectrum is shifted in the positive direction, and the coherent dipole $\pi$ mode is visible at about twice the incoherent tune shift. In the vertical plane, the spectrum is shifted toward negative values, and again the coherent $\pi$ mode is shifted twice as much as the incoherent spectrum.

The simulation results are easily interpreted. Since the transverse distance between two bunches in the long range collision is much larger than the rms beam size, the effects are similar to the coherent interaction of rigid pointlike bunches, and the coherent tune shifts due to parasitic crossings are approximately

$$
\begin{gathered}
\Delta \nu_{\pi}=2(\text { incoherent tune shift) }, \\
\Delta \nu_{\sigma}=0 .
\end{gathered}
$$

For beam separations larger than $\approx 1.5 \sigma$, the slopes of the beam-beam force versus the transverse amplitude are of opposite sign in the two transverse directions, which explains the opposite sign of the tune shifts. 

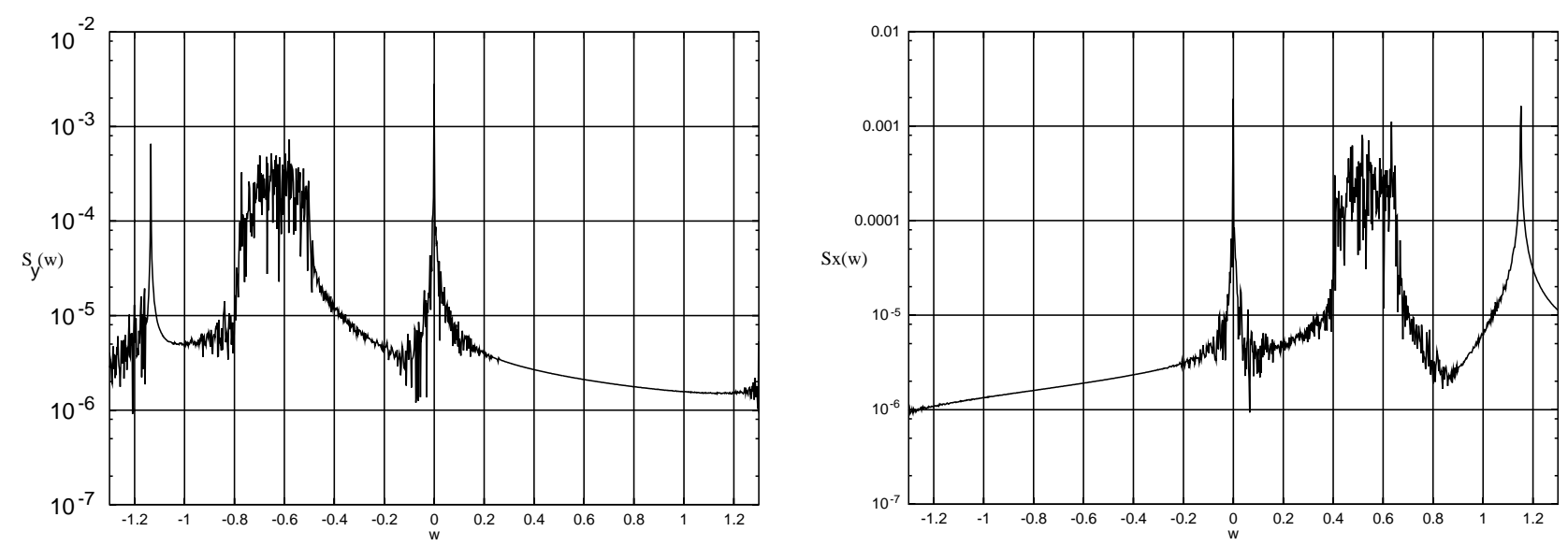

FIG. 9. Spectrum of the vertical (left) and horizontal (right) centroid motion for a long-range collision with horizontal separation $L_{x}=7.5$ (in units of $\sigma_{x}$ ) and no head-on collision (calculated over $2^{17}$ turns with $N=10^{3}$ macroparticles). The horizontal axis gives the tune distance to the unperturbed tune $Q$ in units of $\xi: w=(\nu-Q) / \xi$. The vertical axis gives the oscillation amplitude on a logarithmic scale. The tune shifts due to long-range collisions are of opposite direction in the two transverse planes. The frequency of the coherent $\pi$ mode is at twice the incoherent tune shift.

\section{B. Head-on collisions with alternating crossing}

Next we simulate the combined effect of head-on and long-range interactions. Since the tune shifts from longrange collisions have opposite signs in the two transverse planes, an alternating crossing scheme was proposed for the LHC [16], where the beams are separated in orthogonal planes at the two main IPs. This reduces the overall incoherent tune shift and tune spread by cancellation of the tune shift between IPs. Also the coherent-mode frequency shifts caused by the long-range collisions should be much reduced.

We consider two closely spaced bunches per beam, and two interaction regions. The bunches in beam 1

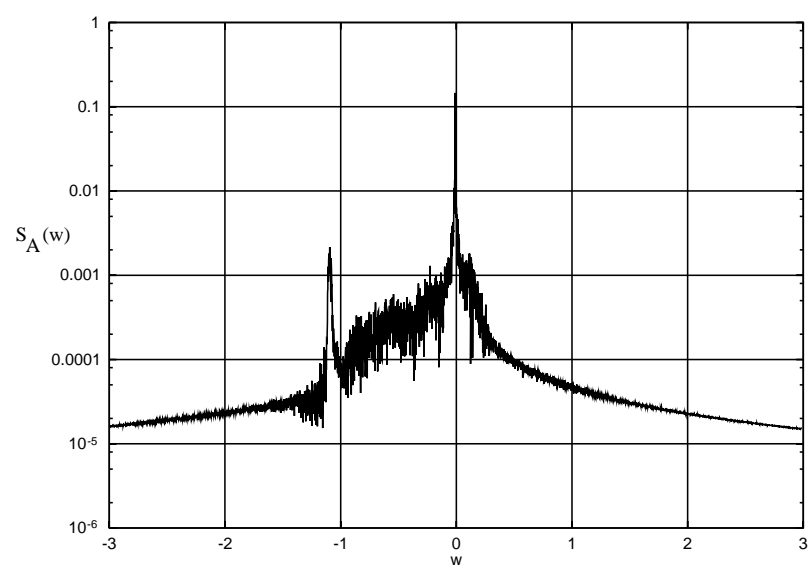

FIG. 10. Spectrum in the case of head-on and long-range collisions with alternating crossing when each bunch collides head-on at two interaction points, and undergoes long-range collisions with $n_{\mathrm{par}}=32$ bunches behind each IP. The horizontal axis is the tune distance to the unperturbed betatron frequency in units of the new incoherent tune shift $2 \xi: w=$ $(\nu-Q) / 2 \xi$. Tracking was performed for $2^{17}$ turns using $N=10^{4}$ macroparticles. are denoted as $a$ and $b$, and those in beam 2 as $c$ and $d$. First the bunches are collided head-on (for instance $a-c$ and $b-d$ ). We then apply a phase advance of $90^{\circ}$ to reach the long-range collision region. There the bunch pairs $(a-d)$ and $(b-c)$ are collided with a horizontal separation of $L_{x}$ and a beam-beam parameter which is $n_{\text {par }}$ times stronger than for the primary collision, representing the accumulated effect of $n_{\text {par }}=32$ parasitic collisions around each IP.

Subsequently, we advance the phase of the beams to reach the other interaction region and evaluate the headon collisions $(a-d)$ and $(b-d)$. This is followed by another phase advance of $90^{\circ}$ to the long-range collision point, where again long-range collisions of the pairs $(a-d)$ and $(b-c)$ are applied, but this time with a vertical separation of $L_{y}$.

The spectrum of the bunch motion is illustrated in Fig. 10. Coherent modes still survive outside the continuum. Collision schemes with and without alternating crossing were compared in Ref. [13], with similar conclusions.

\section{SEPARATED TUNES}

Hofmann [6] pointed out that the coherent frequency shifts can be reduced by separating the tunes of the two beams [17]. The case of equal tunes for both beams is well known and leads to $\sigma$ and $\pi$ modes. Separating the tunes can decouple the coherent motion of the two beams, so that the beams oscillate independently. In the LHC, with two independent rings, this could easily be realized, choosing two of the three proposed working points [18].

We simulate this situation for the head-on case with beam 1 at a tune $Q^{(1)}=0.32$ and beam 2 at a tune $Q^{(2)}=$ 0.31 . An initial offset of \pm 0.1 (in units of $\sigma_{x}$ ) is applied to each beam. Figure 11 shows that the continuum 

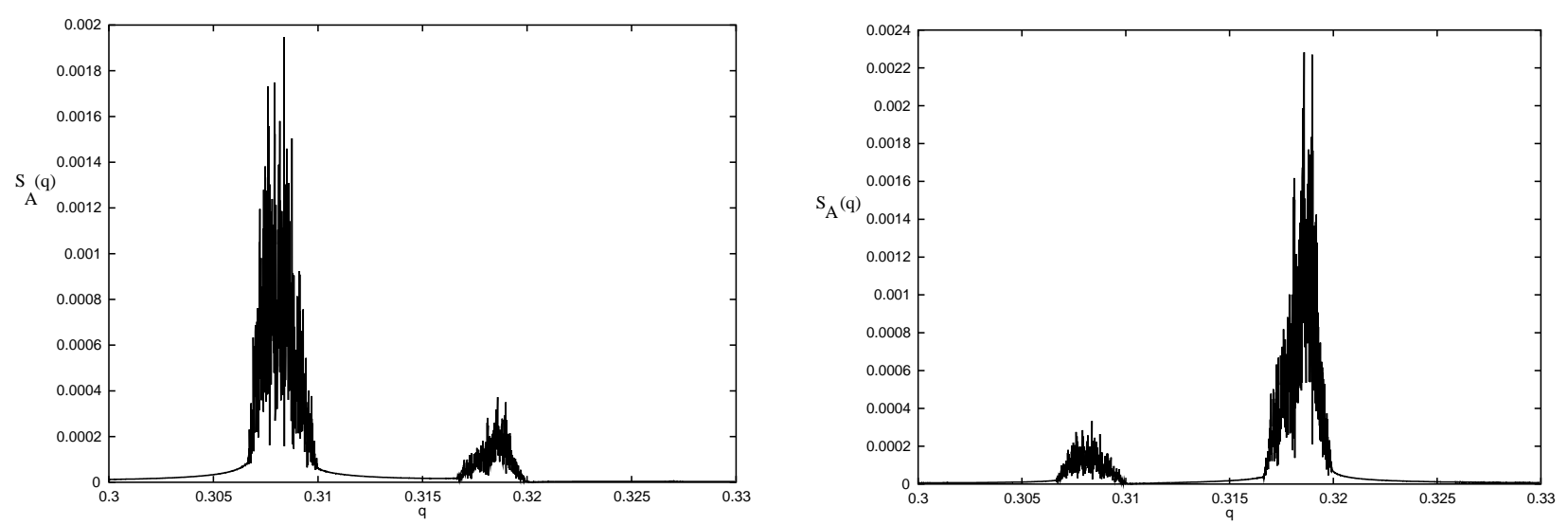

FIG. 11. Beam 2 (left) and beam 1 (right) spectrum of centroid oscillation for head-on collisions with unequal tunes $\left(Q^{(1)}=0.32\right.$, $\left.Q^{(2)}=0.31\right)$ and initial offset $\pm 0.1\left(\sigma_{x}\right),\left(2^{17}\right.$ turns, $N=10^{3}$ macroparticles). Along the horizontal axis we plot the tune, and along the vertical axis the corresponding FFT amplitude. The new coupling modes are inside the continuum and are Landau damped.

of beam 2 extends from $(0.31-\xi=0.3066)$ to 0.31 , and that of beam 1 from $(0.32-\xi)=0.3166$ to 0.32 . Both coupling modes are now inside the incoherent spread of one or the other beam, and Landau damping is restored. For example, the continuum of beam 2 absorbs the energy of the lower-frequency coupling mode, leading to an intrinsic emittance increase of beam 2. The same is true for beam 1, which absorbs the energy of the higher- frequency coupling mode. Now we do not find any narrow peaks corresponding to coherent modes, and we observe only a finite emittance growth.

The new coherent-mode frequencies can be calculated analytically with a simple model of two coupled oscillators with frequencies $Q^{(1)}$ and $Q^{(2)}$ (notice that this model does not include the Yokoya factor). The frequencies of the two eigenmodes are $[6,17]$

$$
\begin{aligned}
Q_{a, b}^{2}= & \frac{\left(Q^{(1)}\right)^{2}+\left(Q^{(2)}\right)^{2}}{2}+\frac{(-\xi)\left(Q^{(1)}+Q^{(2)}\right)}{2} \\
& \pm \frac{1}{2} \sqrt{\left[\left(Q^{(1)}\right)^{2}-\left(Q^{(2)}\right)^{2}\right]^{2}+2(-\xi)\left(Q^{(1)}+Q^{(2)}\right)\left(Q^{(1)}-Q^{(2)}\right)^{2}+(-\xi)^{2}\left(Q^{(1)}+Q^{(2)}\right)^{2}} .
\end{aligned}
$$

As the unperturbed tunes move apart, the eigenmode frequencies $Q_{a}$ and $Q_{b}$ become more and more associated with the tunes of the two individual beams, approaching $Q_{1}-\xi / 2.0$ and $Q_{2}-\xi / 2.0$. As a numerical example, for $Q^{(1)}=0.32$ and $Q^{(2)}=0.31$ the coupled mode frequencies are at $Q_{a}=0.3186$ and $Q_{b}=0.3080\left(Q_{a}\right.$ inside the continuum of beam 1 and $Q_{b}$ inside the continuum of beam 2). The situation is similar when we include long-range collisions [13].

\section{IMPEDANCE}

External impedances cause additional coherent tune shifts. These could push the $\pi$-mode frequency away from the incoherent spectrum and result in loss of Landau damping even when the tunes of the two beams are separated.

We model the effect of the ring impedance in an approximate manner. At every turn we apply a localized kick that depends linearly on the bunch centroid position:

$$
\begin{gathered}
\Delta v_{x}=-4 \pi \Delta Q_{Z, x}\langle x\rangle, \\
\Delta v_{y}=-4 \pi \Delta Q_{Z, y}\langle y\rangle .
\end{gathered}
$$

This results in a coherent tune shift of the centroid motion (dipole mode), but has no effect on the tunes of individual particles (incoherent spectrum). We have simulated cases with negligible horizontal tune shift $\left(\Delta Q_{Z, x}=0\right)$ and different values of the vertical tune shift $\Delta Q_{Z, y}$. For increasing magnitude of the coherent tune shift, we observe the emergence of coherent modes from the incoherent spectrum, as illustrated in Fig. 12.

To preserve the Landau damping established by tune separation, the additional impedance tune shift should be smaller than the distance between the coherent mode frequencies (without impedance) and the continuum boundary, which, for the case of sufficiently unequal tunes, is roughly $\xi / 2.0 \approx 15 \times 10^{-4}$. The total effective LHC impedance at $7 \mathrm{TeV}$ has been estimated to be about $\beta_{\mathrm{av}} \operatorname{Im}\left(Z_{T}\right)_{\text {eff }}=718 \mathrm{M} \Omega$ [19], this gives rise to a coherent tune shift of $\Delta \nu=$ $-I_{b} R /\left(2 E / e 4 \sigma_{s}\right) \beta_{\mathrm{av}} Z \approx-1.3 \times 10^{-4}$. It is expected that this estimate will be at least doubled when other machine components are taken into account. Therefore we can assume for the LHC at top energy an impedanceinduced tune shift of the order of $\Delta \nu \approx-2.5 \times 10^{-4}$ (about $10 \%$ of the beam-beam parameter). This is small 

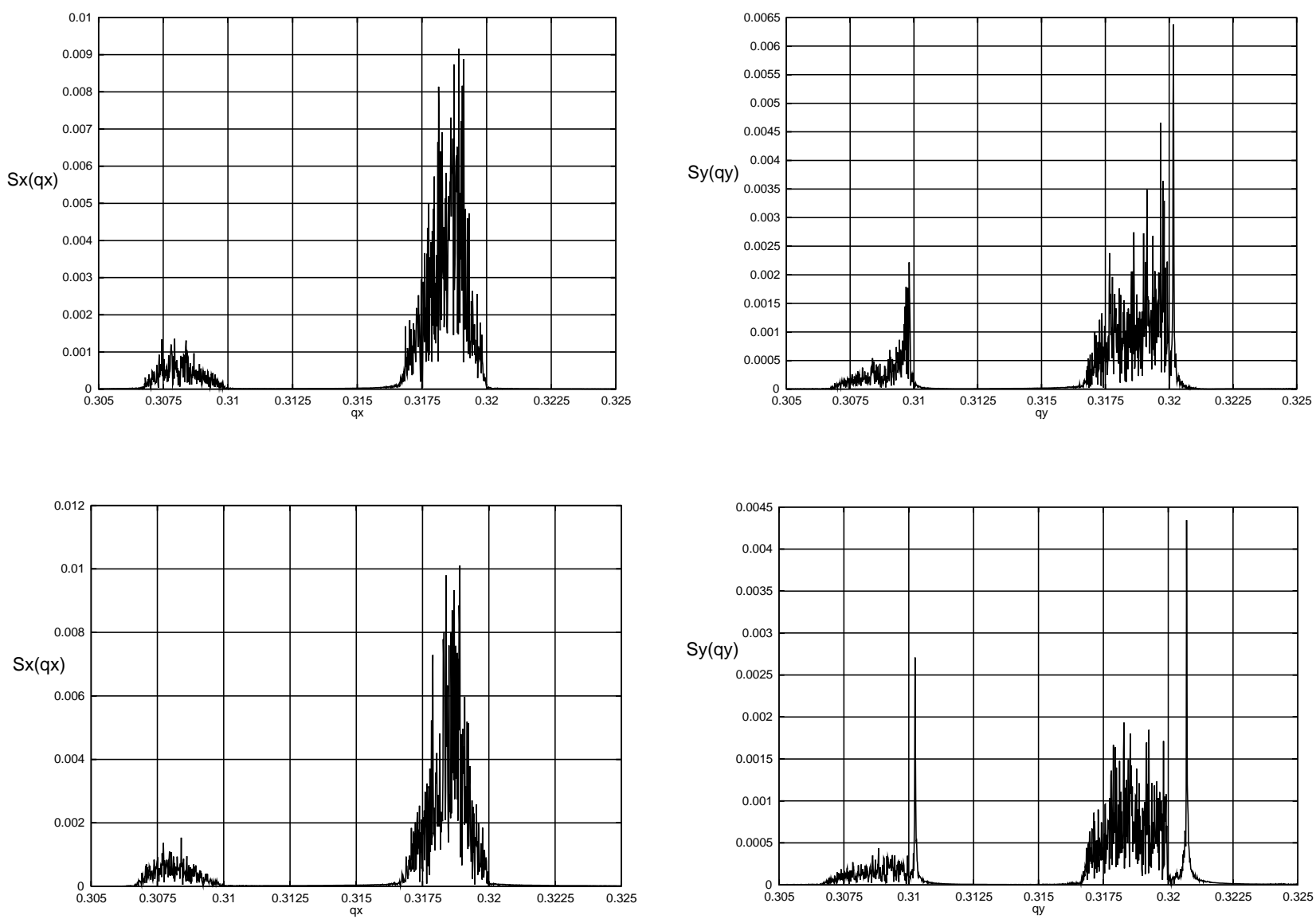

FIG. 12. Two-dimensional strong-strong simulation of the collision of bunch 1 with tune $Q_{x, y}^{(1)}=0.32$ and bunch 2 with tune $Q_{x, y}^{(2)}=0.31$ for $\xi=0.0034$. Along the horizontal axis the tune is plotted, and along the vertical axis is the corresponding oscillation amplitude for the horizontal (left) and vertical (right) motion of beam 1. Top: an additional vertical coherent tune shift $\Delta Q_{Z, y}$ of $+14 \times 10^{-4}$, which pushes one of the vertical coherent modes away from the continuum. Bottom: a vertical coherent tune shift of $+20 \times 10^{-4}$; now both vertical coherent modes are outside of the continuum.

enough that the coherent mode frequencies stay inside the continuum, for the tunes chosen here with a factor of 4 safety margin, and for sufficiently separated tunes with a factor of 6 margin.

\section{CONCLUSIONS}

Using a simplified multiparticle simulation, we have studied the coupled coherent beam-beam modes at the LHC.

For the case of head-on collisions the energy partition between continuum, $\sigma$ and $\pi$ modes after an initial transverse offset agrees well with theoretical predictions [4]. We have also confirmed another prediction [4] that, for equal beam sizes and current ratio $0<r \leq 0.6$, the $\pi$ mode lies within the continuum and is Landau damped. Its energy is transferred to the continuum, leading to an irreversible finite emittance growth. For equal beam-beam parameters of the two beams, we find a $\pi$-mode tune shift of -1.1 in units of $\xi$, sufficiently large to place it outside of the continuum and to lose Landau damping. A $50 \%$ unevenness in the beam size restores Landau damping even for a beam-beam parameter ratio as large as 0.8 .

In the case of two equally strong beams with head-on and long-range collisions, coherent modes exist outside of the continuum, even with alternating crossing at two IPs. In general, these modes are not Landau damped. However, if the betatron tunes of the two beams are sufficiently different, the frequencies of the coherent modes are shifted toward the continuum of one or the other beam and Landau damping can be restored.

Impedances give rise to additional coherent tune shifts. For the expected coherent tune shift at top energy of $-2.5 \times 10^{-4}$, Landau damping is still retained with some safety margin.

In conclusion, there are many scenarios where Landau damping of the coherent beam-beam modes may be lost, but, according to our simulations, separating the tunes of the two rings will stabilize the colliding LHC beams. 


\section{ACKNOWLEDGMENTS}

We thank Y. Alexahin, D. Brandt, M. A. Furman, W. Herr, E. Keil, L. Vos, and F. Ruggiero for stimulating discussions and helpful suggestions.

[1] K. Yokoya and, H. Koiso, Part. Accel. 27, 181-186 (1990).

[2] A. W. Chao and R. D. Ruth, SLAC Report No. SLAC/AP37, 1984.

[3] Proceedings of the Workshop on Beam-Beam Effects in Large Hadron Colliders, Geneva, 1999, edited by J. Poole and F. Zimmermann (CERN Report No. CERN-SL-99039 AP, 1999).

[4] Y. I. Alexahin, Part. Accel. 59, 43 (1999).

[5] A. Hofmann and O. Brüning, in Proceedings of the Workshop on Beam-Beam Effects in Large Hadron Colliders, Geneva, 1999 (Ref. [3]).

[6] A. Hofmann, in Proceedings of the Workshop on BeamBeam Effects in Large Hadron Colliders, Geneva, 1999 (Ref. [3]).

[7] M. Bassetti and G. A. Erskine, CERN Report No. CERNISR-TH/80-06, 1980.

[8] M. Abramowitz and I. A. Stegun, Handbook of Mathemat- ical Functions (Dover, New York, 1965), p. 297.

[9] E. Keil, Nucl. Instrum. Methods Phys. Res. 188, 9-14 (1981).

[10] S. Matsumoto and K. Hirata, KEK Report No. 92-146, 1992.

[11] W. Herr, CERN Report No. CERN-SL/91-34 (AP), LHC Note No. 165, 1991.

[12] R.E. Meller and R.H. Siemann, IEEE Trans. Nucl. Sci. 28, No. 3, 2431-2433 (1981).

[13] M. P. Zorzano and F. Zimmermann, LHC Report No. 314, 1999.

[14] E. Keil, CERN-LEP-NOTE-294, 1981.

[15] L. Vos (private communication).

[16] W. Herr, CERN Report No. CERN SL/90-06 (AP), 1990.

[17] The SPEAR Group, R.H. Helm, M.J. Lee, M. Matera, P. L. Morton, J. M. Paterson, B. Richter, A.P. Sabersky, H. Wiedemann, P. B. Wilson, M. A. Allen, A. E. Augustin, and G. E. Fischer, in Proceedings of the IXth International Conference on High Energy Accelerators, 1974 (SLAC, Stanford, CA, 1974), p. 66.

[18] J. Gareyte, in Proceedings of the Workshop on BeamBeam Effects in Large Hadron Colliders, Geneva, 1999 (Ref. [3]).

[19] F. Ruggiero, CERN Report No. CERN SL/95-09 (AP), LHC Note No. 313, 1995. 